\title{
Finsler Geometry on Higher Order Tensor Fields and Applications to High Angular Resolution Diffusion Imaging
}

\author{
Laura Astola • Luc Florack
}

Published online: 31 August 2010

(c) The Author(s) 2010. This article is published with open access at Springerlink.com

\begin{abstract}
We study 3D-multidirectional images, using Finsler geometry. The application considered here is in medical image analysis, specifically in High Angular Resolution Diffusion Imaging (HARDI) (Tuch et al. in Magn. Reson. Med. 48(6):1358-1372, 2004) of the brain. The goal is to reveal the architecture of the neural fibers in brain white matter. To the variety of existing techniques, we wish to add novel approaches that exploit differential geometry and tensor calculus.

In Diffusion Tensor Imaging (DTI), the diffusion of water is modeled by a symmetric positive definite second order tensor, leading naturally to a Riemannian geometric framework. A limitation is that it is based on the assumption that there exists a single dominant direction of fibers restricting the thermal motion of water molecules. Using HARDI data and higher order tensor models, we can extract multiple relevant directions, and Finsler geometry provides the natural geometric generalization appropriate for multi-fiber analysis. In this paper we provide an exact criterion to determine whether a spherical function satisfies the strong convexity criterion essential for a Finsler norm. We also show a novel fiber tracking method in Finsler setting. Our model incorporates a scale parameter, which can be beneficial in view of the noisy nature of the data. We demonstrate our methods on analytic as well as simulated and real HARDI data.
\end{abstract}

\section{Astola $(\bowtie) \cdot$ L. Florack}

Department of Mathematics and Computer Science, Eindhoven University of Technology, PO Box 513, 5600 MB Eindhoven,

The Netherlands

e-mail: 1.j.astola@tue.nl

L. Florack

e-mail: 1.m.j.florack@tue.nl
Keywords Finsler geometry · Higher order tensors · High Angular Resolution Diffusion Imaging (HARDI)

\section{Introduction}

Diffusion Weighted Imaging (DWI) is a non-invasive medical imaging modality that measures the attenuation of Magnetic Resonance Imaging (MRI) signal due to thermal motion of water molecules. High Angular Resolution Diffusion Imaging (HARDI) is a collective name for techniques to analyze a dense sample of diffusion weighted measurements, typically ranging from 50 to 200 angular directions. It is assumed that the motion of water molecules reveals relevant information of the underlying tissue architecture. The so-called apparent diffusion coefficient $D(g)$, is computed from the signal $S(g)$ using the Stejskal and Tanner (1965) formula

$\frac{S(g)}{S_{0}}=\exp (-b D(g))$

where $g$ is the gradient direction, $S_{0}$ the signal obtained when no diffusion gradient is applied, and $b$ is a parameter associated with the imaging protocol (Mori 2007). This formula assumes that the diffusion is Gaussian distributed. This is indeed justified in view of the large number of hydrogen nuclei $\left(\approx 10^{18} /\right.$ voxel) and the central limit theorem (Rosner 2006).

In the Diffusion Tensor Imaging framework, (1) is interpreted as

$\frac{S(g)}{S_{0}}=\exp \left(-b g^{T} D g\right)$

with a direction independent $3 \times 3$ two-tensor $D$ describing the probability of directional diffusivity at each voxel. 
A natural way to do geometric analysis on the image, is to use the inverse of the diffusion tensor $D$ as the Riemann metric tensor. The heuristic for this is that large diffusion implies short travel time (distance) for an ensemble of diffusing particles. This approach has been exploited to some extent in the DTI literature (O'Donnell et al. 2002; Lenglet et al. 2004; Astola et al. 2007; Astola and Florack 2008; Fuster et al. 2009). Since HARDI data typically contains more directional measurements than DTI, it requires a model that has richer directional information than a local position dependent inner product i.e. Riemannian metric.

Higher order tensor representations (Özarslan and Mareci 2003; Barmpoutis et al. 2007; Florack and Balmashnova 2008) form an interesting alternative to the popular spherical harmonic representation of HARDI data and this is especially suited for a Finsler geometric approach. Finsler geometry has already been considered for HARDI analysis in Melonakos et al. (2008), where the necessary homogeneity condition is imposed on the Finsler norm by definition. In Melonakos et al. (2008) a third power of a ratio $\frac{S}{F R\left(S / S_{0}\right)}$ of the signal $S$ and the Funk-Radon transform of the attenuation $F R\left(S / S_{0}\right)$ is used as the cost function, and the optimal paths that minimize geodesic distances w.r.t. this cost function are computed using dynamic programming.

In this paper a different approach is proposed, using higher order monomial tensors to approximate the spherical functions that represent the complex diffusion/fiber orientation profiles, such as the orientation distribution function (ODF), and constructing a homogeneous Finsler norm from these. Fibers are not modeled here as geodesics. For example the subcortical fibers, having high curvature but modest anisotropy, cannot be modeled as geodesics neither w.r.t. the Riemann metric $\left(D^{-1}\right)$ nor the Finsler norm we propose here. Instead, the streamline tractography, commonly used in DTI, is generalized here to the HARDI case.

This paper is organized as follows. Section 2 contains a very short introduction to Finsler geometry and in Sect. 3 we verify that indeed HARDI measurements can be modeled with a Finsler-structure and give a specific condition which ensures this. In Sect. 4 an explicit method to transform a polynomial tensor presentation, which allows LaplaceBeltrami smoothing, to a monomial one convenient for constructing a Finsler-norm is given. In Sect. 5 we show some results of Finsler fiber-tracking on simulated data sets as well as on two human brain HARDI data. In the appendix we show the details of computing the new strong convexity criterion.

\section{Finsler Geometry}

In a perfectly homogeneous and isotropic medium, geometry is Euclidean, and shortest paths are straight lines.
In an inhomogeneous space, geometry is Riemannian and the shortest paths are geodesics induced by the Levi-Civita connection (Carmo 1993). The Levi-Civita connection is a set of rules that tells one how to take derivatives on a Riemannian manifold. If the connection is linear, symmetric and satisfies the product (Leibniz) rule, then it is unique.

If a medium is not only inhomogeneous, but also anisotropic, ${ }^{1}$ i.e. it has an innate directional structure, the appropriate geometry is Finslerian (Bao et al. 2000; Shen 2001) and the shortest paths are correspondingly Finslergeodesics. As a consequence of the anisotropy, the metric tensor (to be defined below) depends on both position and direction. This is also a natural model for high angular resolution diffusion images.

Another way to look at the difference of Riemann and Finsler geometry is that while Riemannian distances are defined by a position dependent inner product, Finslerian distances are defined by a direction dependent inner product, computed from a position dependent norm. Since the level sets determined by norms can be more complex than those (ellipsoidal) given by an inner product, this approach is suitable for modeling complex diffusion profiles.

Definition 1 Let $M$ be a differentiable manifold and $T_{x} M$ be a (Euclidean) tangent space (approximating the manifold at each point) at $x \in M$. Let $T M=\bigcup_{x \in M} T_{x} M$ be the collection of tangent spaces. Take $y$ to be a vector with positive length in $T_{x} M$. A Finsler norm is a function $F: T M \rightarrow$ $[0, \infty)$ that satisfies each of the following criteria:

1. Differentiability: $F(x, y)$ is $C^{\infty}$ on $T M$.

2. Homogeneity: $F(x, \lambda y)=\lambda F(x, y)$.

3. Strong convexity: The Finsler metric tensor, derived from the Finsler norm, with components

$$
g_{i j}(x, y)=\frac{1}{2} \frac{\partial^{2} F^{2}(x, y)}{\partial y^{i} \partial y^{j}}
$$

is positive definite at every point $(x, y)$ of $T M$.

Note that if $F(x, y)=\sqrt{g_{i j}(x) y^{i} y^{j}}$, then $g_{i j}(x, y)=g_{i j}(x)$ i.e. it reduces to a Riemannian metric.

\section{Finsler Norm on HARDI Higher Order Tensor Fields}

We want to show that higher order tensors, such as those fitted to HARDI data, do define a Finsler norm, which can

\footnotetext{
${ }^{1}$ We will call a medium isotropic if it is endowed with a direction independent inner product, or Riemannian metric. In the literature such a medium is also often referred to as anisotropic due to the directional bias of the metric itself.
} 
be used in the geometric analysis of this data. We take as a point of departure a given orientation distribution function (ODF), which if normalized, is a probability density function on the sphere. Such a spherical function, assigning unit vectors a probability that it coincides with fiber- or diffusion orientations, can be computed from HARDI data by using one of the methods described in the literature (Tuch 2004; Jansons and Alexander 2003; Özarslan et al. 2006; Jian et al. 2007; Descoteaux et al. 2006). The ODF is assumed to be symmetric w.r.t. the origin i.e. an even order function. A higher order symmetric tensor restricted to the sphere, can be defined as follows. Let $\mathbf{y}$ be a unit vector

$$
\begin{aligned}
\mathbf{y}(\theta, \varphi) & =(u(\theta, \varphi), v(\theta, \varphi), w(\theta, \varphi)) \\
& =(\sin \theta \cos \varphi, \sin \theta \sin \varphi, \cos \theta) .
\end{aligned}
$$

It is well known that every even order real spherical polynomial up to order $\ell$ can be represented as a linear combination of the spherical harmonics basis

$Y_{0}^{0}(\theta, \varphi), Y_{2}^{-2}(\theta, \varphi), \ldots, Y_{\ell}^{\ell-1}(\theta, \varphi), Y_{\ell}^{\ell}(\theta, \varphi)$.

In tensor formulation we use the following basis (omitting the arguments $(\theta, \varphi))$

$u^{\ell}, u^{\ell-1} v^{1}, u^{\ell-1} w^{1}, \ldots, u^{p} v^{q} w^{r}, \ldots, w^{\ell}$

where $p+q+r=\ell$. For simplicity we consider fourth order tensors $(\ell=4)$ in what follows, but any even order tensor can be treated in similar way.

We denote a fourth order symmetric tensor (using the Einstein summation convention, i.e. $a_{i} b^{i}=\sum_{i} a_{i} b^{i}$ ) as,

$T(x, \mathbf{y})=T_{i j k l}(x) \mathbf{y}^{i} \mathbf{y}^{j} \mathbf{y}^{k} \mathbf{y}^{l}$.

We fit such a tensor to the ODF-data, using linear least squares method, following Özarslan and Mareci (2003). Suppose we obtain measurements $m_{1}, m_{2}, \ldots, m_{N}$ in $N$ different directions. First we recall that the number of distinct $n$th order monomials of 3 variables $(u, v$ and $w)$ is

$\frac{(3+n-1) !}{(3-1) ! n !}$.

Thus a fourth order symmetric tensor is completely determined by 15 coefficients. We denote the multinomial coefficient as

$\mu(\hat{u}, \hat{v}, \hat{w})=\frac{(\hat{u}+\hat{v}+\hat{w}) !}{(\hat{u} ! \hat{v} ! \hat{w} !)}$,

where $\hat{u}+\hat{v}+\hat{w}=4$ and $\hat{u}$ denotes the multiplicity of component $u$ etc. We omit zero multiplicities from notation $\mu(0, \hat{v}, 0)=\mu(\hat{v})$. To fit tensor (7) to data, first the following $(N \times 15)$ matrix $M$ is constructed:

$$
\left(\begin{array}{cccccc}
\mu(4) u_{1}^{4} & \mu(3,1) u_{1}^{3} v_{1} & \ldots & \mu(2,2) v_{1}^{2} w_{1}^{2} & \ldots & \mu(4) w_{1}^{4} \\
\mu(4) u_{2}^{4} & \mu(3,1) u_{2}^{3} v_{2} & \ldots & \mu(2,2) v_{2}^{2} w_{2}^{2} & \ldots & \mu(4) w_{2}^{4} \\
\vdots & \vdots & & & \vdots & \vdots \\
\mu(4) u_{N}^{4} & \mu(3,1) u_{N}^{3} v_{N} & \ldots & \mu(2,2) v_{N}^{2} w_{N}^{2} & \ldots & \mu(4) w_{N}^{4}
\end{array}\right),
$$

where $u_{i}=u\left(\theta_{i}, \varphi_{i}\right), v_{i}=v\left(\theta_{i}, \varphi_{i}\right)$, etc. corresponding to the $i$ th measurement. Setting the unknown tensor coefficients to be $d_{1}, d_{2}, d_{3}, \ldots, d_{15}$ and defining vectors

$d=\left(\begin{array}{c}d_{1} \\ d_{2} \\ \vdots \\ d_{15}\end{array}\right) \quad$ and $\quad m=\left(\begin{array}{c}m_{1} \\ m_{2} \\ \vdots \\ m_{N}\end{array}\right)$,

we can solve $d$ from the equation

$M \cdot d=m$,

using the pseudo-inverse.

Next we define a Finsler norm $F(x, y)$ corresponding to fourth order ODF-tensor as

$F(x, y)=\left(T_{i j k l}(x) y^{i} y^{j} y^{k} y^{l}\right)^{1 / 4}$.

A general form for any even order $n$ would be then

$F(x, y)=\left(T_{i_{1} \ldots i_{n}}(x) y^{i_{1}} \cdots y^{i_{n}}\right)^{1 / n}$.

In the following, we verify the necessary criteria for this (13) to be a Finsler norm in Definition 1.

1. Differentiability: The tensor field $T(x, y)$ is continuous in $x$ by linear interpolation between the sample points and differentiable w.r.t. $x$ using Gaussian derivatives. As a probability $T(x, \mathbf{y})$ is assumed to be non-negative, and the differentiability of $F(x, y)$ w.r.t. $x$ follows. The differentiability of $F(x, y)$ in $y$ is obvious from the formula (13).

2. Homogeneity: Indeed for any $\alpha \in \mathbb{R}_{+}, x \in M$

$$
\begin{aligned}
F(x, \alpha \mathbf{y}) & =\left(T_{i j k l}(x) \alpha \mathbf{y}^{i} \alpha \mathbf{y}^{j} \alpha \mathbf{y}^{k} \alpha \mathbf{y}^{l}\right)^{1 / 4} \\
& =\alpha F(x, \mathbf{y}) .
\end{aligned}
$$

3a. Strong convexity for a tensor based norm: Since this is a pointwise criterion, we omit here the argument $x$. We compute explicitly the metric tensor from norm (13)

$$
g_{i j}(y)=\frac{1}{2} \frac{\partial^{2} F^{2}(y)}{\partial y^{i} \partial y^{j}}
$$




$$
\begin{aligned}
= & 3 T^{-1 / 2} T_{i j k l} y^{k} y^{l} \\
& -2 T^{-3 / 2} T_{i a b c} y^{a} y^{b} y^{c} T_{j p q r} y^{p} y^{q} y^{r},
\end{aligned}
$$

where we have abbreviated the scalar sum as $T_{i j k l} y^{i} y^{j} y^{k} y^{l}:=T$. To simplify the expression, we multiply it with a positive scalar $c=T^{3 / 2}$, since it does not affect the positive definiteness. We obtain

$$
\begin{aligned}
c \cdot g_{i j}(y)= & 3 T T_{i j k l} y^{k} y^{l} \\
& -2 T_{i a b c} y^{a} y^{b} y^{c} T_{j p q r} y^{p} y^{q} y^{r} .
\end{aligned}
$$

We define a special product inner product candidate

$$
\langle v, v\rangle_{T}:=T_{i j k l} y^{k} y^{l} v^{i} v^{j} .
$$

Then from (17) we have for an arbitrary vector $v$ :

$$
\begin{aligned}
c \cdot g_{i j}(y) v^{i} v^{j}= & \langle y, y\rangle_{T}\langle v, v\rangle_{T}+2\left(\langle y, y\rangle_{T}\langle v, v\rangle_{T}\right. \\
& \left.-\langle y, v\rangle_{T}\langle y, v\rangle_{T}\right) .
\end{aligned}
$$

In case (18) does define an inner product, the CauchySchwarz inequality says that the last term must be nonnegative. Then we have indeed that

$g_{i j}(y) v^{i} v^{j}>0$.

Thus in case the norm function is a power of an even order tensor, the strong convexity is satisfied if the $\langle,\rangle_{T}$ is positive, since it meets all other conditions for an inner product. As a result the original condition for positive definiteness

$$
\begin{aligned}
g_{i j}(y) v^{i} v^{j}= & \left(T^{-1 / 2} T_{i j k l} y^{k} y^{l}\right. \\
& \left.-2 T^{-3 / 2} T_{i a b c} y^{a} y^{b} y^{c} T_{j p q r} y^{p} y^{q} y^{r}\right) v^{i} v^{j} \\
> & 0
\end{aligned}
$$

is reduced to a simpler condition

$T_{i j k l} y^{k} y^{l} v^{i} v^{j}>0$.

3b. Strong convexity for a general norm $F$ : We now state a strong convexity criterion for a general Finsler norm $F(x, y)$ in $\mathbb{R}^{3}$, by analogy to the $\mathbb{R}^{2}$-criterion by Bao et al. (2000). We have put the derivation of the condition into Appendix, and merely state the result here. We consider the so-called indicatrix of the norm function $F(x, y)$ at any fixed $x$, which is the set of vectors $\mathbf{v}(\theta, \varphi)$

$\left\{\mathbf{v} \in \mathbb{R}^{3} \mid F(\mathbf{v})=1\right\}$.

The indicatrix forms a closed surface which is a unit sphere w.r.t. norm function $F$. When $F$ is the Euclidean length the indicatrix becomes the regular sphere. For brevity, we write

$\mathbf{v}:=\mathbf{v}(\theta, \varphi)$.
We denote $\mathbf{v}_{\theta}:=\frac{\partial}{\partial \theta}(\mathbf{v}), \mathbf{v}_{\theta \theta}:=\frac{\partial^{2}}{\partial \theta^{2}}(\mathbf{v})$ and similarly for $\varphi$. We define the following three matrices:

$m=\left(\begin{array}{ccc}\mathbf{v}^{1} & \mathbf{v}^{2} & \mathbf{v}^{3} \\ \mathbf{v}_{\theta}^{1} & \mathbf{v}_{\theta}^{2} & \mathbf{v}_{\theta}^{3} \\ \mathbf{v}_{\varphi}^{1} & \mathbf{v}_{\varphi}^{2} & \mathbf{v}_{\varphi}^{3}\end{array}\right)$,

$m_{\theta}=\left(\begin{array}{ccc}\mathbf{v}_{\theta \theta}^{1} & \mathbf{v}_{\theta \theta}^{2} & \mathbf{v}_{\theta \theta}^{3} \\ \mathbf{v}_{\theta}^{1} & \mathbf{v}_{\theta}^{2} & \mathbf{v}_{\theta}^{3} \\ \mathbf{v}_{\varphi}^{1} & \mathbf{v}_{\varphi}^{2} & \mathbf{v}_{\varphi}^{3}\end{array}\right)$,

$m_{\varphi}=\left(\begin{array}{ccc}\mathbf{v}_{\varphi \varphi}^{1} & \mathbf{v}_{\varphi \varphi}^{2} & \mathbf{v}_{\varphi \varphi}^{3} \\ \mathbf{v}_{\theta}^{1} & \mathbf{v}_{\theta}^{2} & \mathbf{v}_{\theta}^{3} \\ \mathbf{v}_{\varphi}^{1} & \mathbf{v}_{\varphi}^{2} & \mathbf{v}_{\varphi}^{3}\end{array}\right)$.

Then the strong convexity requires:

$\frac{\operatorname{det}\left(m_{\theta}\right)}{\operatorname{det}(m)}<0 \quad$ and $\quad \frac{\operatorname{det}\left(m_{\varphi}\right)}{\operatorname{det}(m)}<-\frac{\left(g_{i j} \mathbf{v}_{\theta}^{i} \mathbf{v}_{\varphi}^{j}\right)^{2}}{g_{i j} \mathbf{v}_{\theta}^{i} \mathbf{v}_{\theta}^{j}}$

a proof of which is provided in Appendix.

The goal of this section was to define Finsler metric tensors $g_{i j}(x, y)$ corresponding to a given tensorial ODF-field. Following a Finsler approach, instead of one metric tensor per voxel one obtains a collection of metric tensors at any $x$. For an illustration, see Fig. 1.

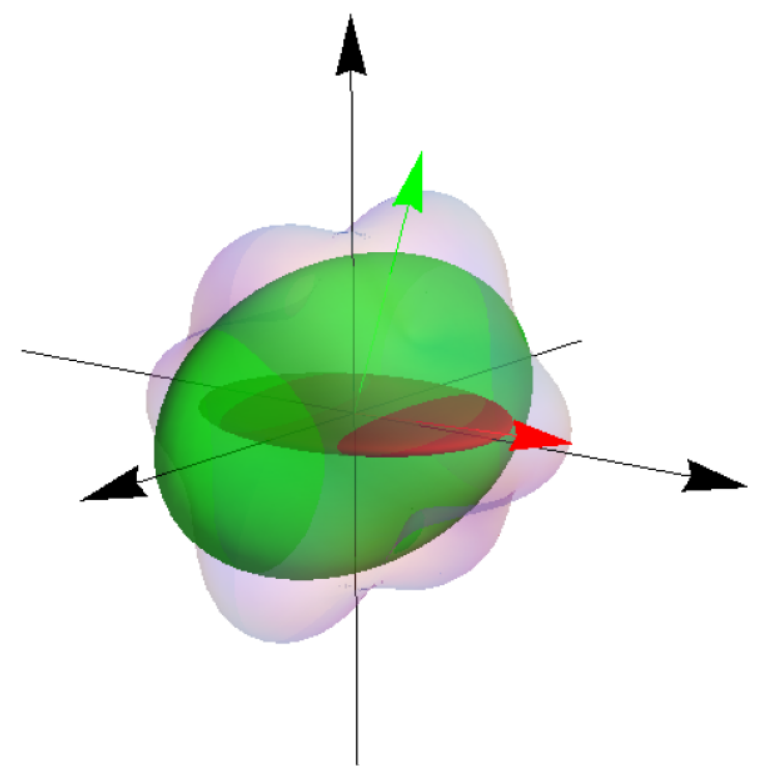

Fig. 1 (Color online) A fourth order spherical tensor (the light purple colored blob), representing an ODF and 2 ellipsoids illustrating the local second order diffusion tensors corresponding to the 2 vectors. The red ellipsoid is determined by the red vector and similarly for the green ellipsoid 


\section{Transforming a Polynomial Tensor to a Monomial Tensor}

Say we wish to regularize our spherical data, and that we wish to use a tensorial representation of the data instead of spherical harmonics. In Florack (2008), a scale space extension for a spherical signal is considered which is a generalization of the Tikhonov regularization. This scale space takes particularly simple form when applied to spherical harmonics (Bulow 2004). Conveniently, in Florack and Balmashnova (2008) it is shown that this regularization technique can also be applied to tensorial case requiring only that the tensor representation is constructed in a hierarchical way, such that the higher order components contain only "residual" information, that can not be contained in lower order terms. In practice this means that to a given ODF, one first fits a zeroth order tensor, and to the residual one fits a second order tensor. Further, one fits iteratively higher order tensors to the residuals until the desired order is reached (for details see Florack and Balmashnova 2008). However, as can be seen from the formulae below, it is not immediately clear how to homogenize a hierarchically expressed tensor, where higher order terms can have negative values.

Thus for Finsler analysis, it is favorable to work with a tensor representation of monomial form

$T(x, \mathbf{y})=T_{i_{1} \cdots i_{n}}(x) \mathbf{y}^{i_{1}} \cdots \mathbf{y}^{i_{n}}$,

and not with an equivalent polynomial expression

$T(x, \mathbf{y})=\sum_{k=0}^{n} \tilde{T}_{i_{1} \cdots i_{k}}(x) \mathbf{y}^{i_{1}} \cdots \mathbf{y}^{i_{k}}$

but to still benefit from the pointwise regularization scheme of the latter:

$T(x, \mathbf{y})_{\tau}=\sum_{k=0}^{n} e^{-\tau k(k+1)} \tilde{T}_{i_{1} \cdots i_{k}}(x) \mathbf{y}^{i_{1}} \cdots \mathbf{y}^{i_{k}}$,

where $\tau$ is the regularization parameter.

Indeed, we can transform a polynomial expression to a monomial one using the fact that our polynomials are restricted to the sphere (4), thus we may expand a $n$th order tensor to a $n+2$ order one and symmetrize it. Symmetrization of a tensor amounts to a projection to the subspace of symmetric tensors (Yokonuma 1992). For an even order (2n) symmetric tensor $T$ the multilinear mapping

$T: \underbrace{\mathbb{S}^{2} \times \cdots \times \mathbb{S}^{2}}_{2 n \text { times }} \rightarrow \mathbb{R}, \quad T(\mathbf{y})=T_{i_{1} \ldots i_{2 n}} \mathbf{y}^{i_{1}} \cdots \mathbf{y}^{i_{2 n}}$,

can be equivalently expressed as a quadratic form

$\tilde{T}_{\alpha \beta} \tilde{\mathbf{y}}^{\alpha} \tilde{\mathbf{y}}^{\beta}$ where $\alpha, \beta=1, \ldots, 3^{n}$. Here $\tilde{T}$ is a square matrix with components

$\tilde{T}_{\alpha \beta}=T_{\sigma_{\alpha}\left(i_{1}\right) \cdots \sigma_{\alpha}\left(i_{n}\right) \sigma_{\beta}\left(i_{1}\right) \cdots \sigma_{\beta}\left(i_{n}\right)}$,

and

$\tilde{\mathbf{y}}^{\alpha}=\mathbf{y}^{\sigma_{\alpha}\left(i_{1}\right)} \cdots \mathbf{y}^{\sigma_{\alpha}\left(i_{n}\right)}$,

where $\sigma_{1}, \ldots, \sigma_{3^{n}}$ are the permutations of components of $\mathbf{y}$ corresponding to each term of the outer product $\mathbf{y} \otimes_{1} \cdots \otimes_{n} \mathbf{y}$.

As an example to clarify the equivalence of (30) and (31), let us consider a spherical fourth order tensor $A_{i j k l}$ in dimension two, with input vectors

$\mathbf{y}=\left(\mathbf{y}^{1}, \mathbf{y}^{2}\right)=(u(\varphi), v(\varphi))=(\cos \varphi, \sin \varphi)$.

We observe that since the tensor is symmetric, a sum

$A_{i j k l} \mathbf{y}^{i} \mathbf{y}^{j} \mathbf{y}^{k} \mathbf{y}^{l}$

is equal to an inner product (omitting the argument $\varphi$ here)

$$
\begin{gathered}
\left(\begin{array}{llllll}
u^{2} & u v & v u & v^{2}
\end{array}\right)\left(\begin{array}{llll}
A_{1111} & A_{1112} & A_{1211} & A_{1212} \\
A_{1111} & A_{1112} & A_{1211} & A_{1212} \\
A_{2111} & A_{2112} & A_{2211} & A_{2212} \\
A_{2121} & A_{2122} & A_{2221} & A_{222}
\end{array}\right) \\
\quad \times\left(\begin{array}{l}
u^{2} \\
u v \\
v u \\
v^{2}
\end{array}\right)
\end{gathered}
$$

In this matrix-form, for example the expansion of a second order tensor with components $T_{i j}$ to a fourth order tensor with components $T_{i j k l}$ can be illustrated in a very simple way in Fig. 2. Such expansion is possible, because the input vectors are unit vectors. For example we can immediately verify the following identity of a zeroth and a second order tensor

$c=\left(\begin{array}{ll}u(\varphi) & v(\varphi)\end{array}\right)\left(\begin{array}{ll}c & 0 \\ 0 & c\end{array}\right)\left(\begin{array}{l}u(\varphi) \\ v(\varphi)\end{array}\right)=c\left(\sin ^{2} \varphi+\cos ^{2} \varphi\right)$.

The same principle extends to dimension three and higher.

A formula for obtaining a fourth order symmetric tensor equivalent to a given second order Cartesian tensor (i.e. that $\left.T_{i j} \mathbf{y}^{i} \mathbf{y}^{j}=T_{k l m n} \mathbf{y}^{k} \mathbf{y}^{l} \mathbf{y}^{m} \mathbf{y}^{n}\right)$ is thus

$T_{i j k l}=\frac{1}{4 !} \sum_{\sigma \in S_{4}} T_{\sigma(i) \sigma(j)} \mathbf{I}_{\sigma(k) \sigma(l)}$

where I is the identity matrix and $S_{4}$ the symmetric group of all permutations of a set of four elements. 

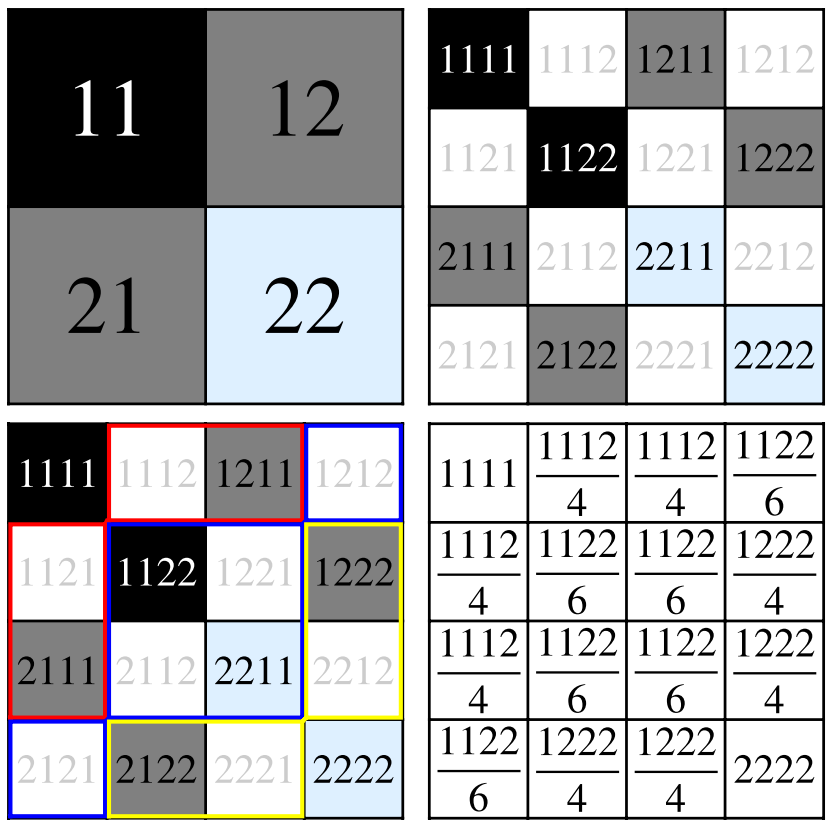

\begin{tabular}{|c|c|c|c|}
\hline 1111 & $\frac{1112}{4}$ & $\frac{1112}{4}$ & $\frac{1122}{6}$ \\
\hline$\frac{1112}{4}$ & $\frac{1122}{6}$ & $\frac{1122}{6}$ & $\frac{1222}{4}$ \\
\hline$\frac{1112}{4}$ & $\frac{1122}{6}$ & $\frac{1122}{6}$ & $\frac{1222}{4}$ \\
\hline$\frac{1122}{6}$ & $\frac{1222}{4}$ & $\frac{1222}{4}$ & 2222 \\
\hline
\end{tabular}

Fig. 2 Top left: A second order symmetric tensor $T$ in 2D. Top right: The Kronecker product of $T$ and the identity matrix $\mathbf{I}$. Here elements with same color have same value (white $=0$ ). Bottom left: The equivalence classes of permutation group $S_{4}$ on sets with elements $\{1,2\}$. Bottom right: To symmetrize the matrix, each element is replaced by the average of all elements in the equivalence class

\section{Fiber Tracking in HARDI Data Using Finsler Geometry}

In DTI setting the most straightforward way of tracking fibers is to follow the principal eigenvector corresponding to the largest eigenvalue of the diffusion tensor until some stopping criterion is reached. When the ODF is modeled as a second order tensor, this straightforward method cannot reveal crossings, since a second order tensor typically has only one principal eigenvector. With higher order ODF model, tracking along principal eigenvectors of Finsler metric tensors, we can do tractography even through crossings. We begin this section by briefly introducing geodesics in Finsler spaces followed by an analytical example, where the optimal paths correspond to geodesics. This is to underline a difference between Finslerian and Riemannian approach. We stress that in general we do not assume fibers to be geodesics in the norm field determined by ODF. In the remaining part of the section some experimental results of Finslertractography is shown both in simulated crossing data as well as in two real HARDI data sets.

\subsection{Geodesics in Finsler Geometry}

Similar to the Riemannian case, in Finsler setting a geodesics $\gamma$ is an extremal path that minimizes the varia- tional length of a curve between fixed endpoints. The EulerLagrange equations of the length integral then give us a local condition according to which an extremal curve has vanishing geodesic curvature (Shen 2001). This amounts to equation

$\ddot{\gamma}^{i}(t)+2 G^{i}(\gamma(t), \dot{\gamma}(t))=0$,

where

$G^{i}(x, y)=\frac{1}{4} g^{i l}(x, y)\left(\frac{\partial F^{2}(x, y)}{\partial x^{k} \partial y^{l}} y^{k}-\frac{\partial F^{2}(x, y)}{\partial x^{l}}\right)$,

is the so-called geodesic coefficient. Using the relationship of the norm and the bilinear form

$g_{i j}(x, y)=\frac{1}{2} \frac{\partial^{2} F^{2}(x, y)}{\partial y^{i} \partial y^{j}}$,

we see that (omitting arguments $x, y$ for brevity)

$$
\begin{aligned}
2 G^{i} & =\frac{1}{2} g^{i l}\left(\frac{\partial}{\partial x^{k}}\left[\frac{\partial}{\partial y^{l}} g_{j n} y^{j} y^{n}\right]-\frac{\partial}{\partial x^{l}}\left[g_{j k} y^{j} y^{k}\right]\right) \\
& =\frac{1}{2} g^{i l}\left(2 \frac{\partial g_{j l}}{\partial x^{k}} y^{j} y^{k}-\frac{\partial g_{j k}}{\partial x^{l}} y^{j} y^{k}\right) \\
& =\frac{1}{2} g^{i l}\left(\frac{\partial g_{j l}}{\partial x^{k}} y^{j} y^{k}+\frac{\partial g_{k l} l}{\partial x^{j}} y^{k} y^{j}-\frac{\partial g_{j k}}{\partial x^{l}} y^{j} y^{k}\right),
\end{aligned}
$$

showing that the geodesic equation in the Finslerian case is formally identical to that in the Riemann geometry

$\ddot{\gamma}^{i}+\Gamma_{j k}^{i} \dot{\gamma}^{j} \dot{\gamma}^{k}=0$,

where $\Gamma_{j k}^{i}$ is the last term in (42). Note that, unlike in the Riemannian case, $\Gamma_{j k}^{i}$ depend on $y$.

\subsection{Analytic Example}

We consider an analytic norm field in $\mathbb{R}^{2}$. From the application point of view we may consider this norm to be the inverse of an ODF. By the inverse we mean the Moebius inversion w.r.t. a sphere. The minima of the norm coincide with the maxima of the ODF and the geodesics (shortest paths) would then coincide with paths of maximal diffusion. Let us take as a convex norm function at each spatial point

$$
\begin{aligned}
F(\varphi) & =(\cos 4 \varphi+4)^{\frac{1}{4}} \\
& =\left(5 \cos ^{4} \varphi+2 \cos ^{2} \varphi \sin ^{2} \varphi+5 \sin ^{4} \varphi\right)^{\frac{1}{4}} .
\end{aligned}
$$

This is an example of fourth order spherical tensor. Such a tensor field could represent a structure/material which has two preferred orientations of diffusion, along which the distance measure is shorter (see Fig. 3). From the fact that $F(\varphi)$ 


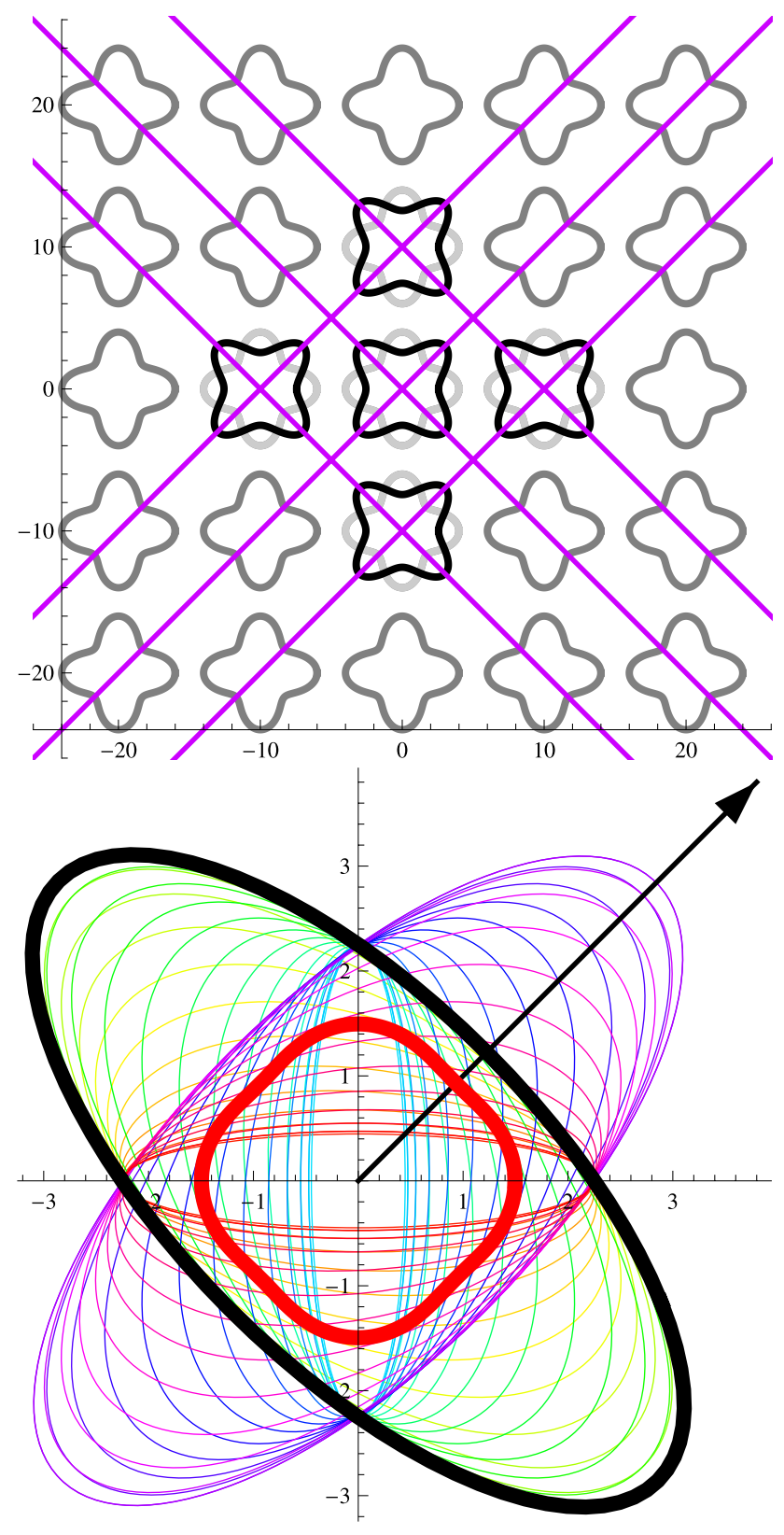

Fig. 3 (Color online) Top: A field of fourth order spherical harmonics $\cos 4 \varphi+4$ representing the norm. In the middle of the figure, the ODF profiles are indicated and some best connected geodesics are drawn. Bottom: 50 ellipses representing metric tensors corresponding to directions $\varphi=\frac{i}{50} 2 \pi(i=1, \ldots, 50)$ of the norm function $F(\varphi)$, and an ellipse with thick boundary corresponding to the metric tensor in direction $\varphi=\frac{\pi}{4}$. The thick red curve is the homogenized norm function $F(\varphi)^{\frac{1}{4}}$

has no $x$-dependence, we conclude that the geodesic coefficients vanish and that the shortest paths connecting any two points coincide with the Euclidean geodesics $\gamma(t)=$ $(t \cdot \cos \varphi, t \cdot \sin \varphi)$, i.e. straight lines. However the so-called connectivity strength of a geodesic (Prados et al. 2006; Astola et al. 2007) is relatively large, only in cases, where the directional norm function is correspondingly small. In Finsler setting the connectivity strength $m(\gamma)$ is:

$m(\gamma)=\frac{\int \sqrt{\delta_{i j} \dot{\gamma}^{i} \dot{\gamma}^{j}} d t}{\int \sqrt{g_{i j}(\gamma, \dot{\gamma}) \dot{\gamma}^{i} \dot{\gamma}^{j}} d t}$,

where the $\delta_{i j}(\gamma)$ is the identity matrix, $\dot{\gamma}(t)$ the tangent to the curve $\gamma(t)$ and $g_{i j}(\gamma, \dot{\gamma})$ the Finsler-metric tensor (which depends not only on the position on the curve but also on the tangent of the curve). For illustration we compute explicitly the metric tensors $g$, using Cartesian coordinates:

$g=\frac{1}{2(4+\cos 4 \varphi)^{3 / 2}}\left(\begin{array}{ll}g_{11} & g_{12} \\ g_{21} & g_{22}\end{array}\right)$,

where

$g_{11}=5(6+3 \cos 2 \varphi+\cos 6 \varphi)$,

$g_{12}=g_{21}=-12 \sin 2 \varphi^{3}$,

$g_{22}=-5(-6+3 \cos 2 \varphi+\cos 6 \varphi)$.

The strong convexity criterion in $\mathbb{R}^{2}$ (Bao et al. 2000) on the indicatrix $\{\mathbf{v}(\varphi) \mid F(\mathbf{v}(\varphi))=1\}$, for metric (46) is satisfied for every $\varphi$, since

$\frac{\ddot{\mathbf{v}}^{1} \dot{\mathbf{v}}^{2}-\dot{\mathbf{v}}^{1} \ddot{\mathbf{v}}^{2}}{\dot{\mathbf{v}}^{1} \mathbf{v}^{2}-\mathbf{v}^{1} \dot{\mathbf{v}}^{2}}=\frac{13-8 \cos 4 \varphi}{(4+\cos 4 \varphi)^{2}}>0$.

The connectivity measure for a (Euclidean) geodesic $\gamma$ can be computed analytically:

$m(\gamma)=\frac{\int d t}{\int(4+\cos (4 \varphi))^{1 / 4} d t}$,

which gives the maximal connectivities in directions

$\frac{\pi}{4}, \frac{3 \pi}{4}, \frac{5 \pi}{4}$, and $\frac{7 \pi}{4}$,

as expected. See Fig. 3 for an illustration. We remark that on this norm field the Riemannian (DTI) framework would similarly result in Euclidean geodesics but the connectivity will be constant over all geodesics, because the second order tensor best approximating the fourth order tensor will be a sphere, thus revealing no information at all of the preferred directions.

\subsection{Finsler Fiber Tracking}

We extend the standard streamline tractography

$$
\left\{\begin{array}{l}
\dot{c}(t)=\underset{|h|=1}{\arg \max }\left\{D_{i j}(c(t)) h^{i} h^{j}\right\}, \\
c(0)=p, \\
\dot{c}(0)=\underset{|h|=1}{\arg \max }\left\{D_{i j}(c(0)) h^{i} h^{j}\right\},
\end{array}\right.
$$


which essentially follows the principal eigenvectors of the diffusion tensors to a Finsler tractography that solves the system

$\left\{\begin{array}{l}\dot{c}(t)=\underset{|h|=1}{\arg \max }\left\{D_{i j}(c(t), \dot{c}(t)) h^{i} h^{j}\right\}, \\ c(0)=p,\end{array}\right.$

where the second order tensor $D_{i j}(c(t), \dot{c}(t))$ is computed from the $n$th order tensor $T_{n}(c(t), \dot{c}(t)$ ) (approximating ODF) as follows

$D_{i j}(c(t), \dot{c}(t))=\left.\frac{1}{2} \frac{\partial^{2}\left(\left(T_{n}(c(t), \mathbf{y})\right)^{1 / n}\right)^{2}}{\partial \mathbf{y}^{i} \partial \mathbf{y}^{j}}\right|_{\mathbf{y}=\dot{c}(t)}$.

The major difference between these two approaches is that the latter depends also on the tangent $\dot{c}(t)$ of the curve. Another difference is that in (51) there is no single initial direction as in (50). Thus the tracking is initially done in many directions, say in directions of all unit vectors of a $n$th order tessellation of the sphere.

The Finsler-fiber tracking can be summarized in the following scheme.

1. Fit a tensor $T_{n}(x, y)$ to the spherical function representing the distribution of fiber orientations (e.g. the ODF).

2. Compute the homogeneous higher order diffusion function $F(x, y)=\left(T_{n}(x, y)\right)^{1 / n}$.

3. Start tracking from point $p$ by computing the $N$ diffusion tensors $D(k)_{k=1, \ldots, N}$ in directions $\mathbf{y}_{1}, \ldots, \mathbf{y}_{N}$, where

$$
D(k)=D_{i j}\left(p, \mathbf{y}_{k}\right)=\left.\frac{1}{2} \frac{\partial^{2}\left(F^{2}(p, y)\right)}{\partial y^{i} \partial y^{j}}\right|_{y=\mathbf{y}_{k}} .
$$

4. Compute the fractional anisotropy (FA) and principal eigenvectors (PE) of diffusion tensors $D(k)$. If the FA is larger than required minimum and $\mathrm{PE}$ in the given angular cone of the tangent vector, take a step in the direction of the PE, otherwise stop.

In the following experiments we have used as an angular condition that the absolute value of the dot product of the principal eigenvector and the unit tangent of the streamline, is greater than 0.6 , and that the FA (of $D_{i j}$ ) is greater than 0.15 , and stepsize is 0.2 voxel dimensions.

\subsection{Simulated Crossing Data}

We show some results of Finsler-tracking in tensor data that simulate crossings. The fourth order tensors in crossings were generated using a multi-tensor model (Frank 2002)

$S=S_{0} \sum_{k=1}^{2} f_{k} e^{-b D_{k}}$,

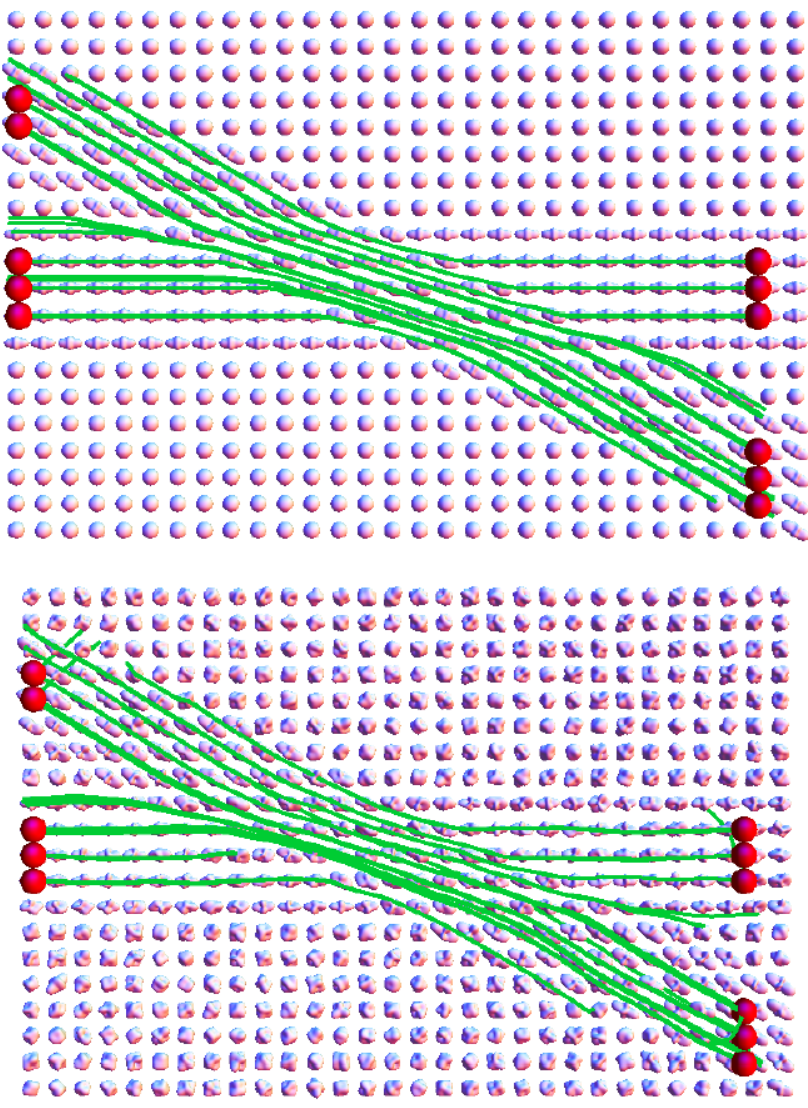

Fig. 4 (Color online) Top: A tensor field simulating fiber bundles crossing at 25 degree angle. Finsler-tracks with initial points marked by red balls. Bottom: Same tensor field with Rician noise $(\mathrm{SNR}=15.3)$ applied. Finsler tracks with same initial points as in the noise-free tensor field

where $f_{k}$ is a weight associated to the $k$ th tensor $D_{k}$ (cf. (1)). In Figs. 4, 6 and 8 we see that Finsler-streamline tracking can indeed resolve crossings. The fibers are well recovered also when Rician noise (the characteristic noise in diffusion MRI) is applied. However, we observe that as the angle between the bundles is narrow, this results in passing/kissing fibers instead of crossings.

\subsection{Real Human Brain Data}

We have computed the Finsler-streamlines in two human brain HARDI data sets. These data sets were acquired with $b$-value 1000 and 132 gradient directions. The data has dimensions $10 \times 104 \times 104$ containing the corpus callosum, but does not contain data below the ventricles. We selected initial points on a horizontal plane, and tracked in 32 directions, which are the first order regular tessellations of a dodecahedron, projected on the circumscribed sphere. For an illustration of subsets of initial points and the corresponding tracks, see Figs. 5 and 7. To distinguish between the fiber orientations, the following color coding was used. If 


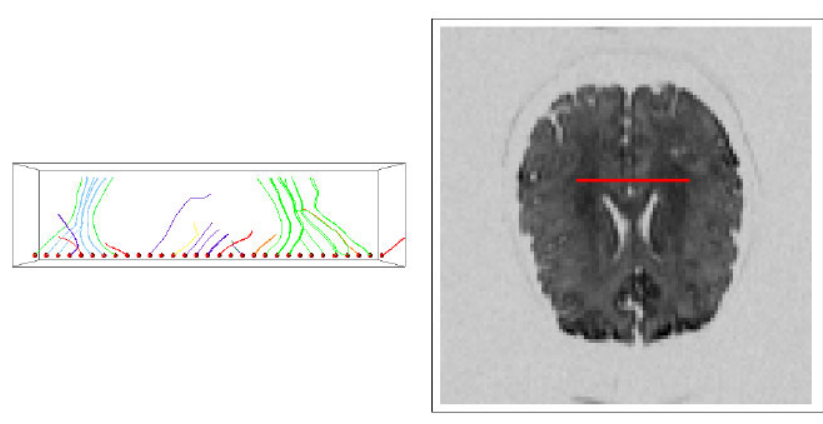

Fig. 5 Left: Finsler-fibers on a subset of initial points. Right: A subset of initial points on a horizontal plane

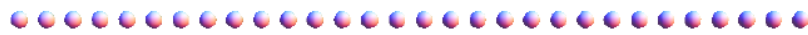

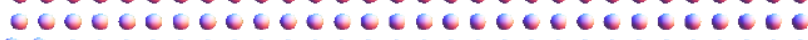

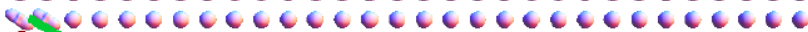

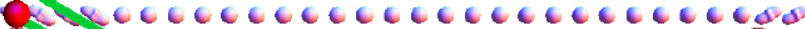

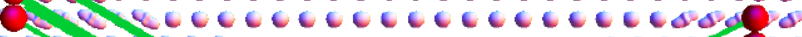

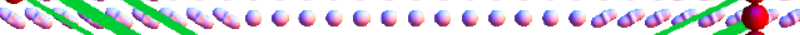

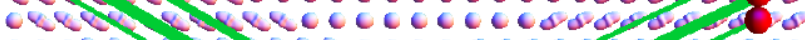

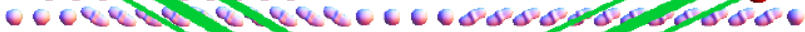

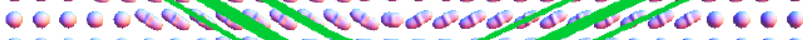

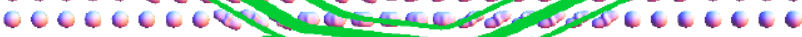

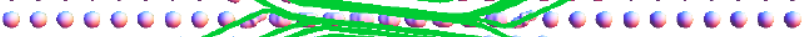

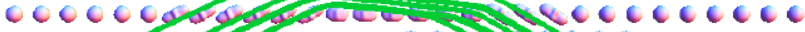

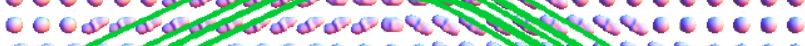

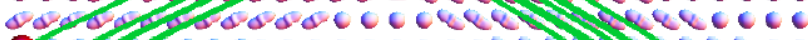

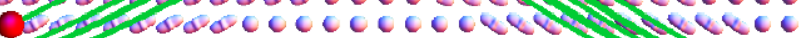
- aradocecececececerban

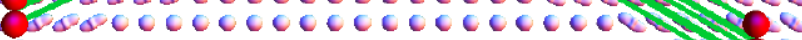

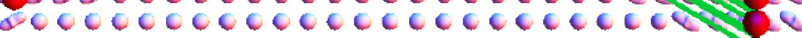

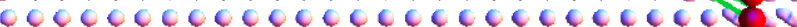

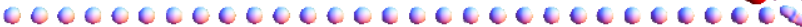

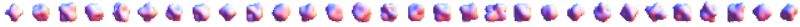

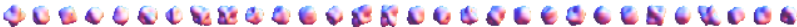

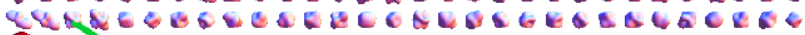

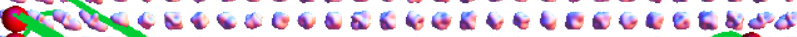

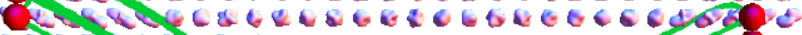

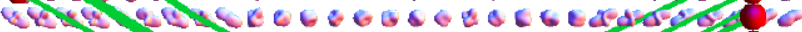

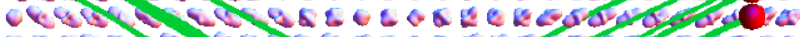
ERSRt

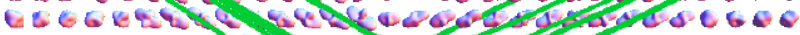

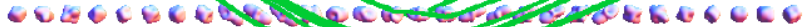

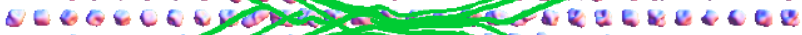

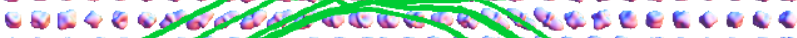

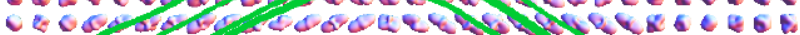

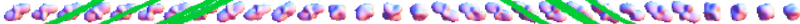

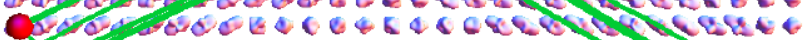

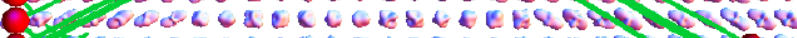

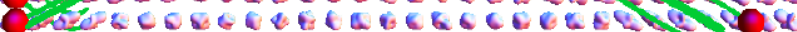

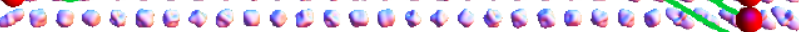

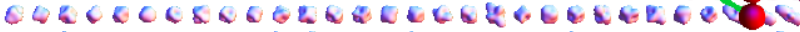

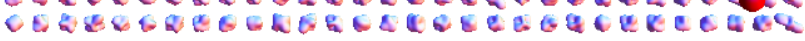

Fig. 6 As in Fig. 4, but with 45 degree angle at the crossing

most of the discretized fiber segments in a curve have orientation closest to "anterior-posterior", the curve is colored yellow. If most of the discretized fiber segments in a curve have orientation closest to "superior-inferior", the curve is colored blue. Similarly curves with major-orientation being "left-right" are colored red. The in-between colors are green between yellow and blue, purple between red and blue and finally orange, between red and yellow. For illustration see Figs. 9 and 10.
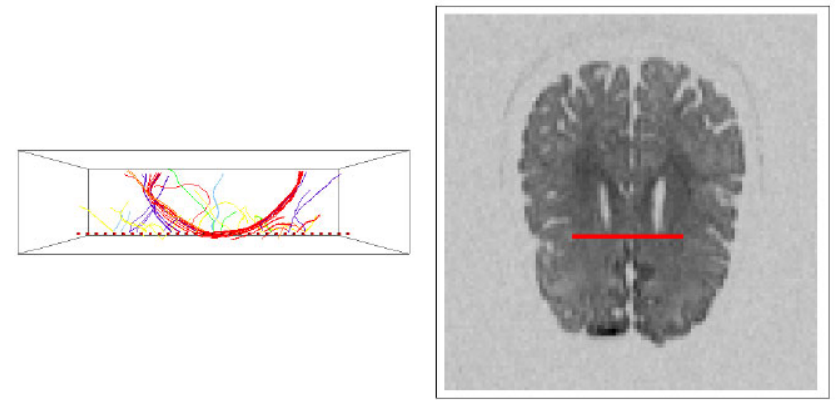

Fig. 7 As in Fig. 5, with another HARDI data
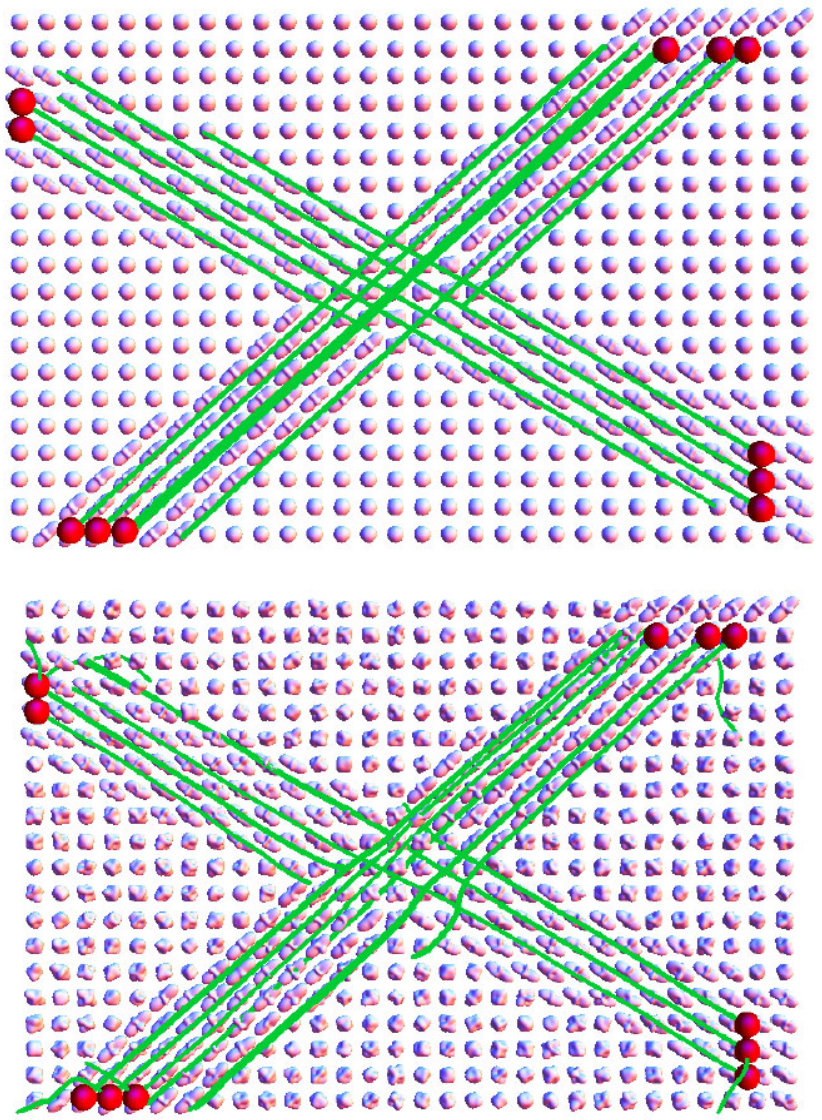

Fig. 8 As in Fig. 4, but with 65 degree angle at the crossing

\section{Conclusions and Future Work}

We have seen that it is indeed possible to analyze spherical tensor fields using Finsler geometry. This gives new methods to analyze the data and is likely to provide new information on the data. For example Finsler diffusion tensors are geometrically well justified local approximations of the more complex tensor describing ODF and Finslerstreamlines can easily propagate through crossings. Another merit of Finsler-streamline tracking is that it does not require any solving of extrema of the ODF and is very simple to implement. 

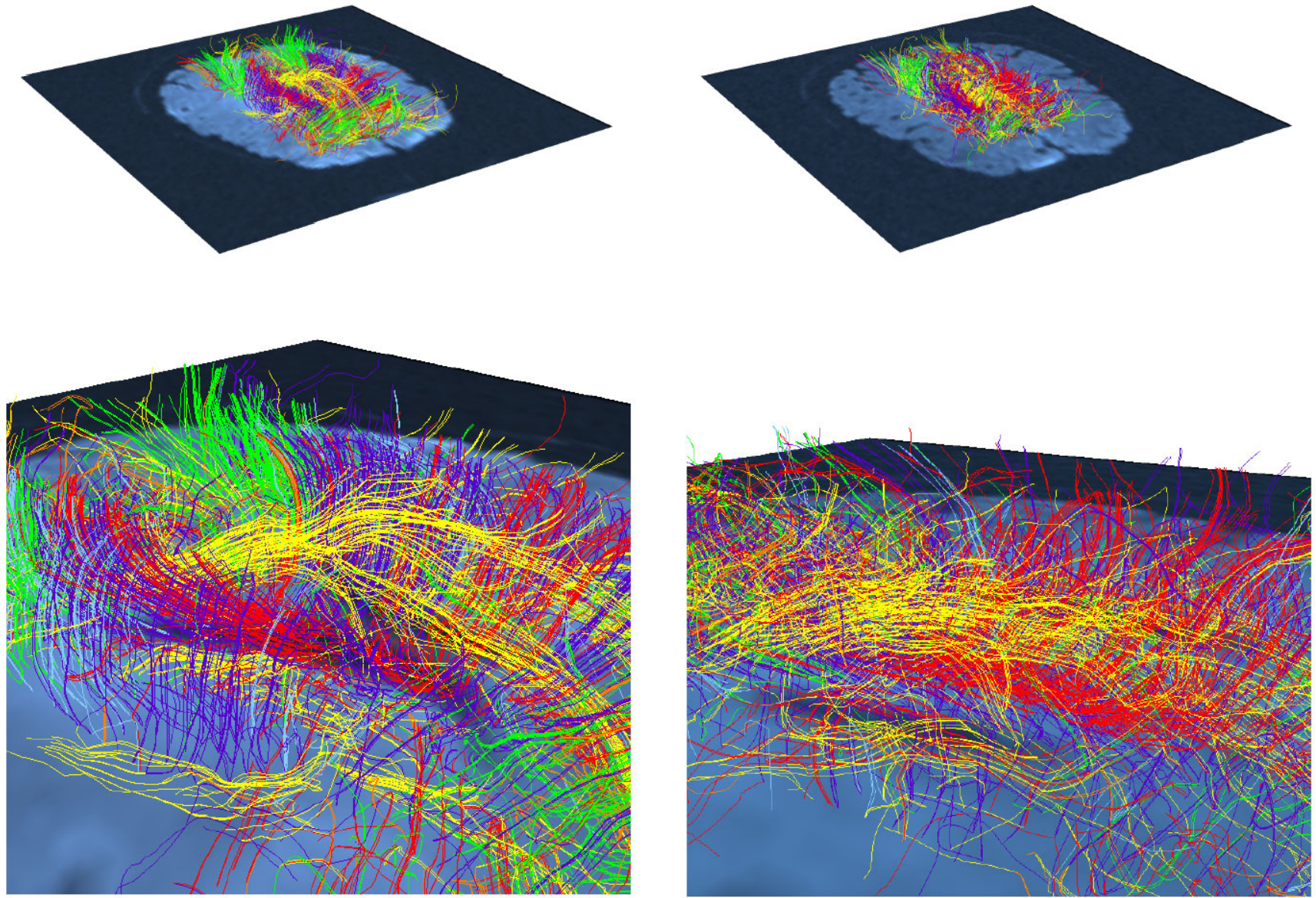

Fig. 9 (Color online) Top: Finsler-fibers on the first set of human brain data. Bottom: A zoom in to see the crossings of the corpus callosum and the corona radiata (red and blue). The yellow fibers resembling the cingulum run over the red fiber layer (the corpus callosum)

Various Finsler curvatures (that vanish in Riemannian space) are examples of geometric quantities which can give new information on the geometry of HARDI signal field and which is an interesting subject for further studies.

Acknowledgements The Netherlands Organization for Scientific Research (NWO) is gratefully acknowledged for financial support. We want to thank Vesna Prckovska and Paulo Rodrigues for kindly providing a program to simulate crossings in HARDI data. We are specially grateful to Evgeniya Balmashnova and Jim Portegies for their valuable advice and to Biomedical Image Analysis group at Eindhoven University of Technology for providing the human brain data.

Open Access This article is distributed under the terms of the Creative Commons Attribution Noncommercial License which permits any noncommercial use, distribution, and reproduction in any medium, provided the original author(s) and source are credited.

\section{Appendix}

We derive the general condition for

$g_{i j}(y) v^{i} v^{j}>0$,

Fig. 10 Top: Finsler-fibers on the second set of human brain data. Bottom: A zoom in to the fibers

to be valid in $\mathbb{R}^{3}\left(=T_{x} M\right)$. From the homogeneity of the norm function $F$, it follows that it is sufficient to have this condition on the unit level set of the norm. We consider this level surface i.e. the set of vectors $\mathbf{v}$ for which $F(\mathbf{v})=1$ and a parametrization

$\mathbf{v}(\theta, \varphi)=\left(\mathbf{v}^{1}(\theta, \varphi), \mathbf{v}^{2}(\theta, \varphi), \mathbf{v}^{3}(\theta, \varphi)\right)$.

In what follows we abbreviate $g_{i j}=g_{i j}(x, y)$. From $F(\mathbf{v})=1$ we have

$g_{i j} \mathbf{v}^{i} \mathbf{v}^{j}=1$.

Taking derivatives of both sides and using a consequence of Euler's theorem for homogeneous functions (Bao et al. 2000, p. 5) that says

$\frac{\partial g_{i j}}{\partial \mathbf{v}^{k}} \mathbf{v}^{k}=0$,

we obtain

$g_{i j} \mathbf{v}_{\theta}^{i} \mathbf{v}^{j}=0$,

$g_{i j} \mathbf{v}_{\varphi}^{i} \mathbf{v}^{j}=0$, 
implying $\mathbf{v}_{\theta} \perp_{g} \mathbf{v}$ and $\mathbf{v}_{\varphi} \perp_{g} \mathbf{v}$.

Taking derivatives once more, we get

$g_{i j} \mathbf{v}_{\theta \theta}^{i} \mathbf{v}^{j}=-g_{i j} \mathbf{v}_{\theta}^{i} \mathbf{v}_{\theta}^{j}$,

$g_{i j} \mathbf{v}_{\varphi \varphi}^{i} \mathbf{v}^{j}=-g_{i j} \mathbf{v}_{\varphi}^{i} \mathbf{v}_{\varphi}^{j}$,

$g_{i j} \mathbf{v}_{\theta \varphi}^{i} \mathbf{v}^{j}=-g_{i j} \mathbf{v}_{\theta}^{i} \mathbf{v}_{\varphi}^{j}$.

We may express an arbitrary vector $\mathbf{u}$ as a linear combination of orthogonal basis vectors:

$\mathbf{u}=\alpha \mathbf{v}+\beta \mathbf{v}_{\theta}+\gamma\left(\mathbf{v}_{\varphi}-\frac{\left\langle\mathbf{v}_{\varphi}, \mathbf{v}_{\theta}\right\rangle}{\left\langle\mathbf{v}_{\theta}, \mathbf{v}_{\theta}\right\rangle} \mathbf{v}_{\theta}\right)$.

We substitute this expression for $\mathbf{u}$ to the left hand side of (55) and obtain:

$$
\begin{aligned}
g_{i j} \mathbf{u}^{i} \mathbf{u}^{j}= & \alpha^{2} g_{i j} \mathbf{v}^{i} \mathbf{v}^{j}-\beta^{2} g_{i j} \mathbf{v}_{\theta \theta}^{i} \mathbf{v}^{j} \\
& -\gamma^{2}\left(g_{i j} \mathbf{v}_{\varphi \varphi}^{i} \mathbf{v}^{j}+\frac{\left(g_{i j} \mathbf{v}_{\theta}^{i} \mathbf{v}_{\varphi}^{j}\right)^{2}}{g_{i j} \mathbf{v}_{\theta}^{i} \mathbf{v}_{\theta}^{j}}\right),
\end{aligned}
$$

because the mixed terms vanish due to the orthogonality of basis vectors.

On the other hand, for v's on the indicatrix we have as a consequence of Euler's theorem on homogeneous functions (denoting $F_{\mathbf{v}^{i}}=\frac{\partial F}{\partial \mathbf{v}^{i}}$ ):

$F_{\mathbf{v}^{i}} \mathbf{v}^{i}=F(\mathbf{v})=1$.

Differentiating (63) w.r.t. $\theta$ and $\varphi$, we obtain two equations:

$F_{\mathbf{v}^{i}} \mathbf{v}_{\theta}^{i}=0$,

$F_{\mathbf{v}^{i}} \mathbf{v}_{\varphi}^{i}=0$,

for $F$ is a homogeneous function.

The matrices $m, m_{\theta}, m_{\varphi}$ are as defined in (25). Solving system of (63), (64) and (65) we get:

$F_{\mathbf{v}^{1}}=-\frac{\mathbf{v}_{\varphi}^{2} \mathbf{v}_{\theta}^{3}-\mathbf{v}_{\varphi}^{3} \mathbf{v}_{\theta}^{2}}{\operatorname{det}(m)}$

$F_{\mathbf{v}^{2}}=-\frac{\mathbf{v}_{\varphi}^{3} \mathbf{v}_{\theta}^{1}-\mathbf{v}_{\varphi}^{1} \mathbf{v}_{\theta}^{3}}{\operatorname{det}(m)}$,

$F_{\mathbf{v}^{3}}=-\frac{\mathbf{v}_{\varphi}^{1} \mathbf{v}_{\theta}^{2}-\mathbf{v}_{\varphi}^{2} \mathbf{v}_{\theta}^{1}}{\operatorname{det}(m)}$.

Now using equalities

$F_{\mathbf{v}^{i}}=g_{i j} \mathbf{v}^{j}, \quad g_{i j} \mathbf{v}_{\theta \theta}^{i} \mathbf{v}^{j}=F_{\mathbf{v}^{k}} \mathbf{v}_{\theta \theta}^{k}$,

$g_{i j} \mathbf{v}_{\varphi \varphi}^{i} \mathbf{v}^{j}=F_{\mathbf{v}^{k}} \mathbf{v}_{\varphi \varphi}^{k}$

and

$g_{i j} \mathbf{v}_{\theta \theta}^{i} \mathbf{v}^{j}=\frac{\operatorname{det}\left(m_{\theta}\right)}{\operatorname{det}(m)}, \quad g_{i j} \mathbf{v}_{\varphi \varphi}^{i} \mathbf{v}^{j}=\frac{\operatorname{det}\left(m_{\varphi}\right)}{\operatorname{det}(m)}$ we obtain

$$
\begin{aligned}
g_{i j} \mathbf{u}^{i} \mathbf{u}^{j}= & \alpha^{2}-\beta^{2} g_{i j} \mathbf{v}_{\theta \theta}^{i} \mathbf{v}^{j} \\
& -\gamma^{2}\left(g_{i j} \mathbf{v}_{\varphi \varphi}^{i} \mathbf{v}^{j}+\frac{\left(g_{i j} \mathbf{v}_{\theta}^{i} \mathbf{v}_{\varphi}^{j}\right)^{2}}{g_{i j} \mathbf{v}_{\theta}^{i} \mathbf{v}_{\theta}^{j}}\right)>0
\end{aligned}
$$

if

$$
\frac{\operatorname{det}\left(m_{\theta}\right)}{\operatorname{det}(m)}<0 \text { and } \frac{\operatorname{det}\left(m_{\varphi}\right)}{\operatorname{det}(m)}<-\frac{\left(g_{i j} \mathbf{v}_{\theta}^{i} \mathbf{v}_{\varphi}^{j}\right)^{2}}{g_{i j} \mathbf{v}_{\theta}^{i} \mathbf{v}_{\theta}^{j}}
$$

\section{References}

Astola, L., \& Florack, L. (2008). Sticky vector fields and other geometric measures on diffusion tensor images. In CVPR: Vol. 20. MMBIA2008, IEEE Computer Society workshop on mathematical methods in biomedical image analysis, held in conjunction with CVPR 2008 (pp. 1-7). Anchorage: Springer.

Astola, L., Florack, L., \& ter Haar Romeny, B. (2007). Measures for pathway analysis in brain white matter using diffusion tensor images. In Information processing in medical imaging: Vol. 20. Information processing in medical imaging: 20th international conference (IPMI 2007) (pp. 642-649). Kerkrade: Springer.

Bao, D., Chern, S. S., \& Shen, Z. (2000). An introduction to RiemannFinsler geometry. Berlin: Springer.

Barmpoutis, A., Jian, B., Vemuri, B., \& Shepherd, T. (2007). Symmetric positive 4th order tensors and their estimation from diffusion weighted MRI. In Information processing in medical imaging: Vol. 20. Information processing in medical imaging: 20th international conference (IPMI 2007) (pp. 308-319). Kerkrade: Springer.

Bulow, T. (2004). Spherical diffusion for 3D surface smoothing. IEEE Transactions on Pattern Analysis and Machine Intelligence, 26(12), 1650-1654.

Carmo, M. P. D. (1993). Riemannian geometry (2nd ed.). Mathematics: theory \& applications. Boston: Birkhäuser.

Descoteaux, M., Angelino, E., Fitzgibbons, S., \& Deriche, R. (2006). Regularized, fast and robust analytical q-ball imaging. Magnetic Resonance in Medicine, 58(3), 497-510.

Florack, L. (2008). Codomain scale space and regularization for high angular resolution diffusion imaging. In CVPR: Vol. 20. CVPR workshop on tensors in image processing and computer vision. Anchorage: Springer.

Florack, L., \& Balmashnova, E. (2008). Decomposition of high angular resolution diffusion images into a sum of self-similar polynomials on the sphere. In Proceedings of the eighteenth international conference on computer graphics and vision, GraphiCon'2008 (pp. 26-31). Moscow, Russian Federation.

Florack, L., \& Balmashnova, E. (2008). Two canonical representations for regularized high angular resolution diffusion imaging. In MICCAI workshop on computational diffusion MRI (pp. 94-105), New York, USA.

Frank, L. (2002). Characterization of anisotropy in high angular resolution diffusion-weighted MRI. Magnetic Resonance in Medicine, 47, 1083-1099.

Fuster, A., Astola, L., \& Florack, L. (2009). A riemannian scalar measure for diffusion tensor images. In Proceedings of 13th international conference on computer analysis of images and patterns. Muenster, Germany.

Jansons, K., \& Alexander, D. (2003). Persistent angular structure: new insights from diffusion magnetic resonance imaging data. Inverse Problems, 19, 1031-1046. 
Jian, B., Vemuri, B., Özarslan, E., Carney, P., \& Mareci, T. (2007). A novel tensor distribution model for the diffusion-weighted MR signal. NeuroImage, 37, 164-176.

Lenglet, C., Deriche, R., \& Faugeras, O. (2004). Inferring white matter geometry from diffusion tensor MRI: application to connectivity mapping. In Proc. eighth European conference on computer vision (ECCV) (pp. 127-140). Prague, Czech Republic.

Melonakos, J., Pichon, E., Angenent, S., \& Tannenbaum, A. (2008). Finsler active contours. IEEE Transactions on Pattern Analysis and Machine Intelligence, 30(3), 412-423.

Mori, S. (2007). Introduction to diffusion tensor imaging. Amsterdam: Elsevier.

O'Donnell, L., Haker, S., \& Westin, C. F. (2002). New approaches to estimation of white matter connectivity in diffusion tensor MRI: elliptic PDEs and geodesics in a tensor-warped space. In LNCS: Vol. 2488. Proc. medical imaging, computing and computer assisted intervention (MICCAI) (pp. 459-466). Berlin: Springer.

Özarslan, E., \& Mareci, T. (2003). Generalized diffusion tensor imaging and analytical relationships between diffusion tensor imaging and high angular resolution diffusion imaging. Magnetic Resonance in Medicine, 50, 955-965.

Özarslan, E., Shepherd, T., Vemuri, B., Blackband, S., \& Mareci, T. (2006). Resolution of complex tissue microarchitecture using the diffusion orientation transform. NeuroImage, 31, 1086-1103.
Prados, E., Soatto, S., Lenglet, C., Pons, J. P., Wotawa, N., Deriche, R., \& Faugeras, O. (2006). Control theory and fast marching techniques for brain connectivity mapping. In Conf. computer vision and pattern recognition (CVPR) (pp. 1076-1083). New York: IEEE Computer Society.

Rosner, B. (2006). Fundamentals of biostatistics (6th ed.) London: Thomson Brooks/Cole

Shen, Z. (2001). Lectures on Finsler geometry. Singapore: World Scientific.

Stejskal, E., \& Tanner, J. (1965). Spin diffusion measurements: spin echoes ion the presence of a time-dependent field gradient. The Journal of Chemical Physics, 42(1), 288-292.

Tuch, D. (2004). Q-ball imaging. Magnetic Resonance in Medicine, 52(4), 577-582.

Tuch, D., Reese, T., Wiegell, M., Makris, N., Belliveau, J., \& van Wedeen, J. (2004). High angular resolution diffusion imaging reveals intravoxel white matter fiber heterogeneity. Magnetic Resonance in Medicine, 48(6), 1358-1372.

Yokonuma, T. (1992). Tensor spaces and exterior algebra (Vol. 108). Providence: American Mathematical Society. 